\title{
The influence of atrial fibrillation on cardiac resynchronization therapy
}

\begin{abstract}
More successful recognition and treatment of cardiovascular risk factors and diseases continues to decrease mortality and increase the proportion of elderly population. Therefore, there are more people with increased risk of developing heart failure and atrial fibrillation (AF) in the course of their lives. The prevalence of AF, in patients with heart failure, increases with the severity of the disease, reaching up to $40 \%$ in advanced cases. In these patients with heart failure, $\mathrm{AF}$ is an independent predictor of morbidity and mortality increasing the risk of death and hospitalization. Despite the excellent results obtained with different drugs, the optimal medical treatment can fail in the intention to improve symptoms and quality of life of patients with severe heart failure. Thus, the necessity to use cardiac devices emerges facing the failure of optimal medical treatment in order to achieve hemodynamic improvement. The simultaneous electric stimulation of both ventricles with Cardiac Resynchronization Therapy (CRT) results in a significant hemodynamic improvement restoring a more homogeneous contraction pattern. However, CRT is interrupted in over $30 \%$ of patients after successful implantation of a CRT device, and the most common reasons for interruption of CRT are the development of AF $(18 \%)$ and loss of left ventricular capture $(10 \%)$. Therefore, there should be a strong effort with different maneuvers to prevent $\mathrm{AF}$, since it would significantly improve the ability to deliver CRT in patients with heart failure. The search for better pharmacological maneuvers should continue to provide the help needed to cardiac devices. The incorporation of the AF suppression algorhythm to CRT devices may be very useful in eliminating AF, allowing a better performance of the CRT device without interruption. Sinus rhythm is associated with a long-term improvement in left ventricular systolic function after AF ablation. Therefore, $\mathrm{AF}$ ablation may have a primary role as a rhythm control strategy in the definite treatment of $\mathrm{AF}$ in patients with $\mathrm{HF}$.
\end{abstract}

Keywords: atrial fibrillation, cardiac resynchronization therapy, atrial vulnerability, abnormal atrial endocardial electrograms
Volume I Issue 2 - 2014

\author{
Osmar Antonio Centurion \\ Department of Cardiology, Asuncion National University, \\ Paraguay
}

\begin{abstract}
Correspondence: Osmar A Centurion, Professor of Medicine, Cardiology Department, Clinic Hospital,Asuncion National University, Trejo Y Sanabria 1657, Sajonia, Asuncion, Paraguay, Tel 595-21-421423, Email osmarcenturion@hotmail.com
\end{abstract}

Received: April 27, 2014 | Published: May 21, 2014

\section{Introduction}

Patients with heart failure are at increased risk of developing atrial fibrillation (AF), despite medical improvements made in recent years. AF can complicate the course of heart failure (HF) leading to acute pulmonary edema. ${ }^{1-3}$ The prevalence of AF, in patients with $\mathrm{HF}$, increases with the severity of the disease, reaching up to $40 \%$ in advanced cases. In these HF patients, AF is an independent predictor of morbidity and mortality increasing the risk of death and hospitalization in $76 \%{ }^{4-6}$ More successful recognition and treatment of cardiovascular risk factors and diseases continues to decrease mortality and increase the proportion of elderly population.

In recent years, we have observed a good number of new drugs that avoid or reverts progression of HF. Nevertheless, despite the excellent results obtained with different drugs, the optimal medical treatment can fail in the intention to improve symptoms and quality of life of patients with severe HF. Thus, the necessity to use cardiac devices emerges facing the failure of optimal medical treatment in order to achieve hemodynamic improvement and correction of the physio-pathological alterations. It was demonstrated that Cardiac Resynchronization Therapy (CRT) reverts the ventricular reverse remodeling produced by chronic HF, and it is suggested that improvement in mechanical synchrony is the predominant mechanism. It has been shown that CRT increases the left ventricular filling time, decreases septal disquinesia and mitral regurgitation, allowing a hemodynamic improvement and better quality of life. ${ }^{7-11}$ These beneficial effects are, apparently dependent on continuous bi-ventricular stimulation since interruption of electric stimulation produce a progressive but not immediate loss of effect. Nevertheless, CRT is interrupted in over one-third of patients after successful implantation of a CRT device, and the most common reason for interruption of CRT is the development of AF. Therefore, this manuscript will review the influence of AF on CRT patients and the different ways to prevent its development.

\section{Electrophysiological properties in atrial fibrillation}

$\mathrm{AF}$ is a common arrhythmia, and its incidence rise sharply with age. The process of aging and its effect on the histhological appearance of the conduction system of the heart have been scantly described. Echocardiographic measurements that were performed in elderly patients confirm the histhological evidence of increasing prevalence of left ventricular wall hypertrophy in aging population. ${ }^{12}$ The resulting left ventricular diastolic dysfunction with aging may increase the size of the left atrium predisposing elderly patients to develop AF. Some investigators have observed that normal histological changes in the atrial muscle occur with advancing age. These include a reduction in the number of myocardial cells within the sinus node, a generalized loss of atrial myocardial fibers in the nearness of the internodal tracts, as well as an increase in the quantity of connective tissue which leads to an apparent loss of myocardial fiber continuity. ${ }^{13-19}$ It was reported that $\mathrm{AF}$ in some aged patients was associated with loss of muscle 
fibers in the sinoatrial node and its approaches without any clear pathological cause, ${ }^{13}$ while others have shown degenerative changes in the conduction system with age. ${ }^{20}$

The increase in prevalence of $\mathrm{AF}$ in older persons has been reported to be associated with degeneration of the atrial muscle in pathological studies. It was demonstrated in well designed studies, that there is clear evidence in the human atrial muscle of age-related electrical uncoupling of the side-to-side connections between bundles, related to the proliferation of extensive collagenous tissue septa in intracellular spaces. ${ }^{21,22}$ The atrial myocardial cells in the elderly appear to be more susceptible to arrhythmias when calcium homeostasis is disturbed and especially under certain conditions that enhance calcium loading. Experimental studies clearly suggest that overload in ionized calcium in the senescent human atrial myocardial cells may play an important role in the development of arrhythmias. ${ }^{23,24}$ Strong evidence of abnormalities of the conduction system in an apparently healthy elderly population has been demonstrated. Prolongation of the PR interval, high prevalence of atrioventricular nodal and His-Purkinje disease, and unexplained sinus node abnormalities were consistently found in older apparently healthy individuals. ${ }^{25-28}$ Muscle loss with advancing age was found to be accompanied by an increase in fibrous tissue in both the sinoatrial node and the internodal tracts. ${ }^{14-16}$ It was strongly suggested that muscle loss and increase of fibrosis in the atria is a slow but continuous process starting around 60 years of age. ${ }^{13}$ Since aging has a profound effect on structural changes of the atrium, and since fractionated atrial endocardial electrograms may reflect nonsynchronised, delayed local electrical activity through a diseased atrial muscle, ${ }^{17}$ it seems reasonable to assume that there may be detectable age induced changes in the electrophysiological properties of the atrial muscle to develop atrial fibrillation (Figure 1)..$^{29,30}$ The precise electrophysiological and patho-physiological bases for AF initiation and maintenance have not been resolved. Since the beginning of this century, the debate on ectopic foci versus re entry as the mechanism underlying atrial fibrillation (AF) in humans has continued. It has been reported that ectopic foci from the pulmonary veins can initiate $\mathrm{AF}$ and can also act as drivers for maintaining AF. However, not all patients with atrial arrhythmias initiate AF. A substrate for atrial propensity to $\mathrm{AF}$ is required for $\mathrm{AF}$ initiation and maintenance. As newer and more sophisticated technology become available, controversies about AF genesis have reemerged, which tells us that there is still a lot to learn about this arrhythmia. New advances may be relevant to the ultimate understanding of the mechanisms of $\mathrm{AF}$ initiation by the interaction of the propagating wavefronts with anatomic or functional obstacles in their paths.

\section{Cardiac resynchronization therapy in the management of HF}

In recent years, we have observed a good number of new drugs that avoid or reverts progression of HF. Nevertheless, despite the excellent results obtained with different drugs, the optimal medical treatment can fail in the intention to improve symptoms and quality of life of patients with severe HF. Thus, the necessity to use cardiac devices emerges facing the failure of optimal medical treatment in order to achieve hemodynamic improvement and correction of the physiopathological alterations. Patients with HF and complete left bundle branch block (LBBB) commonly have an abnormal movement of the interventricular septum that is related with interventricular dissynchrony and the resultant abnormal pressure gradient between the two ventricles. ${ }^{31-33}$ Due to this abnormal septal movement, there is an increase in the end systolic diameter of the left ventricle and a decrease in regional septal ejection fraction. Patients with LBBB with or without cardiac disease may show a decrease global left ventricular ejection fraction, a decrease in cardiac output, and $\mathrm{dP} /$ dt. $^{33,34}$ In addition, in cases of ventricular dissynchrony, the closure of the mitral valve may be incomplete because atrial contraction is not followed by a time-adecuate ventricular systole. If this delay is sufficiently long, a ventriculo-atrial pressure gradient is generated which promotes mitral regurgitation in the early phase of diastole. It is easy to imagine that ventricular dissynchrony in HF patients puts the failing heart in additional mechanical disadvantage. The simultaneous electric stimulation of both ventricles with CRT results in a significant hemodynamic improvement restoring a more homogeneous contraction pattern (Figure 2). CRT can reduce the interventricular and intraventricular mechanical dissynchrony produced by LBBB. It has been shown that CRT increases the left ventricular filling time, decreases septal disquinesia and mitral regurgitation, allowing a hemodynamic improvement (Figures 3\&4). ${ }^{35-37}$ These beneficial hemodynamic changes are already seen in a few days and are followed by chronic adaptations that allow long term benefits. Several longitudinal clinical studies demonstrated beneficial effects of CRT in left ventricular remodeling. There was a structural and functional ventricular improvement during CRT. At 3 months, there was a significant improvement in left ventricular ejection fraction, and a significant decrease in end systolic and end diastolic volumes. ${ }^{38,39}$ These beneficial effects are, apparently dependent on continuous bi-ventricular stimulation since interruption of electric stimulation produce a progressive but not immediate loss of effect. Therefore, CRT reverts the ventricular reverse remodeling produced by chronic $\mathrm{HF}$, and it is suggested that improvement in mechanical synchrony is the predominant mechanism.

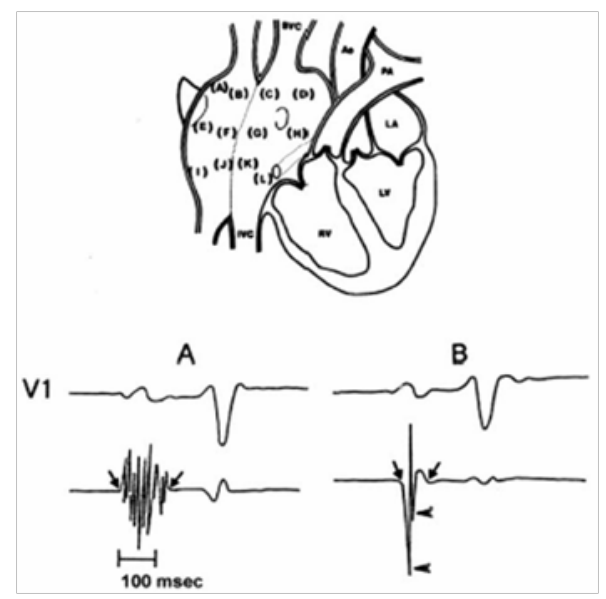

Figure I (Top) Twelve endocardial mapping sites in the right atrium. Atrial endocardial electrograms were recorded in each patient from the anterior lateral, posterior, and medial aspects of the high (a-d), mid (e-h), and low (i-l) right atrium.

(Bottom) (A) Abnormal atrial electrogram with 10 fragmented deflections and $130 \mathrm{~ms}$ in duration versus

(B) Normal atrial electrogram with 2 fragmented deflections and $80 \mathrm{~ms}$ in duration.

Ao: Aorta; IVC: Inferior Vena cava; LA: Left atrium; LV: Left ventricle; PA: Pulmonary artery; RV: Right ventricle; SVC: Superior vena cava

Reproduced with permission from Centurion et al. ${ }^{30}$ 


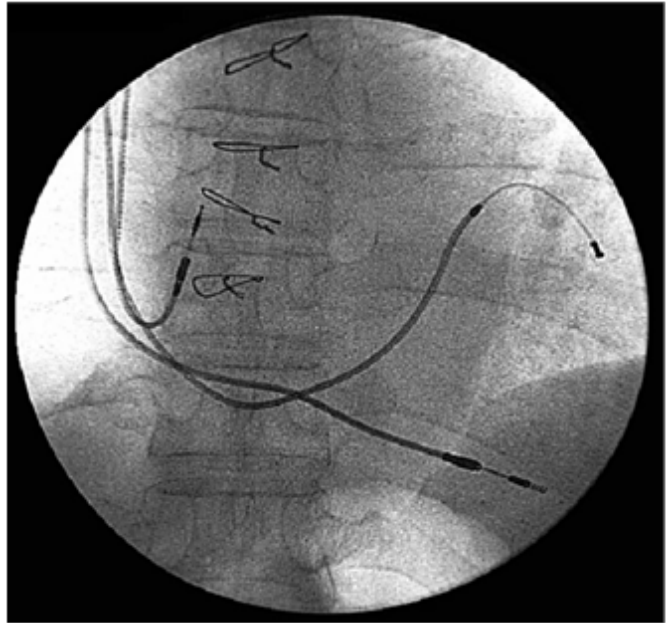

Figure 2 Catheter electrodes position during CRT. By placing pacing electrodes in the coronary sinus, the right ventricular apex, and in the right atrial appendage, CRT can deliver simultaneous electrical stimulation of both ventricles which results in a significant hemodynamic improvement restoring a more homogeneous contraction pattern. Reproduced with permission from Centurion OA. ${ }^{65}$

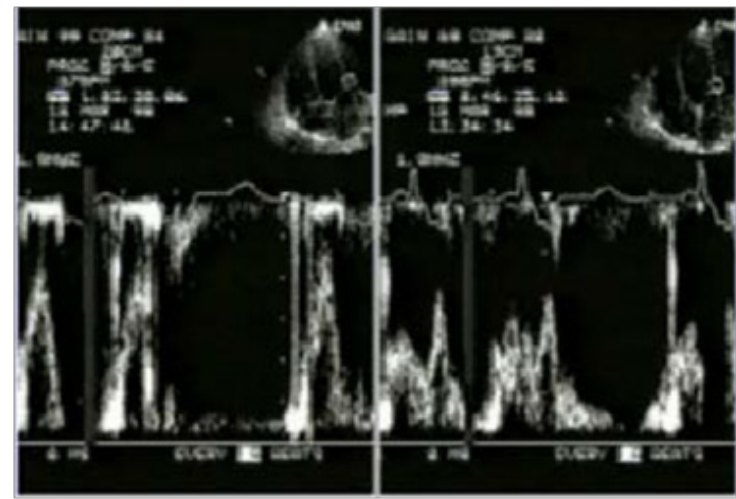

Figure 3 Transmitral Pulse Wave Doppler Echocardiography. With the CRT device on, there is an increase and improvement in the left ventricular filling time. Reproduced with permission from Centurion OA ${ }^{65}$
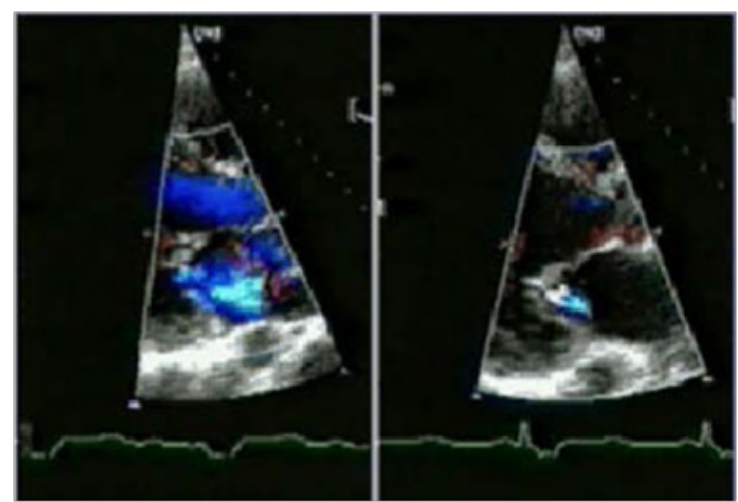

Figure 4 Transmitral Color Doppler Echocardiography with the CRT device on, there is a decrease in mitral regurgitation. Reproduced with permission from Centurion OA. ${ }^{65}$

However, CRT is interrupted in over one-third after successful implantation of a CRT device, and the most common reasons for interruption of CRT are the development of AF (18\%) and loss of left ventricular capture (10\%). However, CRT can be re-instituted in a high proportion of patients so that only $5 \%$ of patients who successfully undergo implantation of a CRT device permanently lose CRT. Almost one fifth of patients who undergo successful implantation of a defibrillator capable of delivering CRT experience an AF with a rapid ventricular response, which at least temporarily results in the inability to deliver CRT.

\section{Pharmacological management of emergent AF in CRT patients}

There is a high prevalence of $\mathrm{AF}$ in the heart failure population. ${ }^{4}$ Since AF is the most common reason for interruption of CRT, it emphasizes the importance of maintaining sinus rhythm in patients treated with CRT devices. ${ }^{40}$ It is not clear whether CRT reduces the incidence of AF in HF patients. Predictors of interruption of CRT as the result of the development of AF in the HF population include a previous history of AF, a relatively slow resting heart rate, and the absence of therapy with both beta-blockers and angiotensin converting enzyme (ACE) inhibitors. ${ }^{40}$ These findings are consistent with an analysis of the SOLVD study which found that treatment with enalapril markedly reduces the risk of development of AF in patients with left ventricular dysfunction ${ }^{41}$. Therefore, although it is not clear whether the use of both beta-blockers and ACE inhibitors directly influence the effectiveness of CRT, their use appears to improve the ability to deliver CRT. The ACE inhibitors produce a decrease in atrial pressure and in left ventricular end diastolic pressure in HF patients. ${ }^{42}$ Therefore, it is possible that these agents could decrease the susceptibility to develop AF simply by decreasing atrial pressure and atrial wall stress and consequently by attenuation of atrial enlargement. However, a decrease in atrial fibrosis was demonstrated experimentally only with ACE inhibitors despite similar decrease in atrial pressure obtained with hidralazine. ${ }^{42}$ Among other potentially beneficial mechanisms of ACE inhibition, a direct anti arrhythmic effect cannot be excluded. Even in the absence of heart failure, it seems that angiotensin II directly contributes to atrial electrical remodeling. The shortening of the atrial refractoriness during rapid atrial pacing is more pronounced in the presence of angiotensin II. However, this electrical change was prevented with a previous treatment with candesartan or captopril..$^{43}$ There was a beneficial effect on $\mathrm{AF}$ recurrence with irbesartan in patients with persistant $\mathrm{AF}$ who underwent electrical cardioversion. ${ }^{44}$ When the drug was administered 3 weeks before cardioversion combined with amiodarone, there was a significant decrease in recurrent episodes of AF. The greater benefit of blocking angiotensine II type I receptors occurred during the first 2 months after electrical cardioversion, suggesting an important role of irbesartan in atrial electrical remodeling after cardioversion. It is very interesting to note that the ACE inhibitor is apparently more effective in patients with lesser symptoms. ${ }^{45}$ This is probably due to potentially reversible milder structural changes in patients with lesser symptoms. Wachtell et al. ${ }^{46}$ demonstrated that losartan reduced the incidence of new onset $\mathrm{AF}$ in 33\% compared to atenolol despite a similar blood pressure control in both treated groups. In addition, the clinical relevance of preventing new onset AF was clearly demonstrated, since AF was associated with a 2 to 5 fold greater cardiovascular morbidity and mortality, cerebrovascular accidents and hospitalization due to heart failure. New onset $\mathrm{AF}$ was reduced $45 \%$ with trandolapril in the TRACE study. ${ }^{47} \mathrm{~A}$ sub-analysis of the SOLVD study showed a $78 \%$ reduction of new onset $\mathrm{AF}$ with enalapril. ${ }^{45}$ It is important to note that both were placebo controlled studies, therefore, it is probable that the antihypertensive effect of enalapril contributed to the less incidence of AF decreasing atrial pressure and left ventricular end diastolic pressure. In this regard, the LIFE study showed that high systolic pressure is an independent predictor of the development of new onset 
AF. ${ }^{46}$ The LIFE study showed that patients with AF history had a reduction of $42 \%$ in combined end point and cardiovascular morbidity and mortality, with a $45 \%$ reduction in the risk of cerebrovascular accidents. ${ }^{48}$ A probable explanation of the benefit obtained with losartan, could be the regression of atrial hypertrophy. Ventricular hypertrophy is an important predictor of the development of new onset AF. Patients with left ventricular hypertrophy have atrial enlargement, which is associated with an increased risk of cerebrovascular accidents in normotensive and hypertensive adults. ${ }^{49-52}$

\section{Atrial pacing algorhythms for AF prevention}

It is clear to see that prevention of AF will improve the ability to deliver CRT. In this regard, it is very useful the atrial fibrillation suppression algorhythm (AFSA) in dual-chamber permanent pacemakers. $^{52}$ Indeed, CRT combined with a refined atrial tachyarrhythmia prevention pacing algorithm appears to be an important addition in the management of AF. The Atrial Dynamic Overdrive Pacing Trial (ADOPT), designed to assess the clinical efficacy and safety of the AF suppression algorithm ${ }^{\mathrm{TM}}$ (St. Jude Medical) in patients with permanent pacemaker with prior history of $\mathrm{AF}$, demonstrated that the overdrive atrial pacing algorithm was safe and decreased the symptomatic AF burden. ${ }^{53}$ However, all patients had normal EF and the primary outcome was symptomatic AF burden. The Management of Atrial fibrillation Suppression in AFHF Comorbidity Therapy (MASCOT) study evaluated whether the addition of atrial overdrive pacing to CRT could reduce the incidence of permanent AF in patients with HF. ${ }^{54-57}$ The overall incidence of permanent $\mathrm{AF}$ was low and was similar for the two treatment groups. It is likely that the advanced atrial remodeling in the setting of HF and AF may preclude benefit from atrial algorithms in this altered milieu. The AFSA is a stimulation parameter designed specifically to suppress AF. It eliminates the unnecessary rapid stimulation produced by the pacemaker associated to the fixed overdrive stimulation when the patient is at rest. It even performs the overdrive stimulation when the intrinsic atrial rate of the patient increases in response to physical activity. It is a valuable tool to apply to paroxysmal and persistent $\mathrm{AF}$ in selected patients that need a permanent pacemaker. ${ }^{52}$ It allows a beneficial reduction of symptoms and costs of the treatment of AF.

The benefits of the atrial fibrillation suppression algorithm are:

1. Automatical suppression of paroxysmal and persistent AF

2. Significant decrease in symptomatic episodes of AF

3. Decrease in the need for painful electric cardioversion

4. Improvement in the quality of life of the patients

5. Effective and safety

6. Well tolerated by $97 \%$ of the patients

The AFSA promotes an atrial stimulation with adequate rates for the patient. It differs from the overdrive pattern procedure with fixed rates that stimulate the heart well beyond the normal resting rates, which can be much slower in physical activities. It dynamically adjusts the stimulation rates in manner that stimulates the heart slightly over the intrinsic atrial rate regardless of the activity status. ${ }^{52}$

\section{Recent findings on rate and rhythm control in AF patients with HF}

$\mathrm{AF}$ and $\mathrm{HF}$ are common cardiovascular pathologies with high comorbidities and mortality and severe prognostic implications. They frequently co-exist accounting for an enormous clinical and economic burden on healthcare. AF patients may have left ventricular dysfunction due to inappropriate increase in heart rate, loss of atrial contraction, irregularity of the heart beat intervals, or due to low cardiac output related to tachycardia-induced cardiomyopathy. ${ }^{58-61}$ Since these deleterious effects on cardiac function may be reversible, it is of most importance to terminate atrial fibrillation and maintain sinus rhythm in these already compromised HF patients. In this context, it is very interesting the findings of several studies. ${ }^{62-64}$ Nedios S et al., ${ }^{62}$ studied $69 \mathrm{AF}$ patients with HF with a mean ejection fraction of $31 \%$ who underwent AF radiofrequency ablation. Most of the patients had persistent AF. $65 \%$ of the patients were free of AF at a mean follow-up of 28 months. They observed an improvement of left ventricular function following AF ablation. In patients who remained in sinus rhythm after AF ablation the ejection fraction improved by $15 \%$ at 6 months and continued to improve reaching a mean of 53\% increase at 28 months. Sinus rhythm, but not hear rate, was found to be an independent predictor of improvement in ejection fraction at 24 months post-AF ablation. The authors concluded that sinus rhythm is associated with a long-term improvement in left ventricular systolic function after AF ablation. The implication is that AF ablation may improve the systolic function in HF patients even when there was already a good heart rate control. On the other hand Sairaku Aet al., ${ }^{64}$ demonstrated an incomplete cure of the tachycardia-induced cardiomyopathy secondary to rapid AF in HF patients by the rate control strategy without sinus conversion. These studies clearly suggest that AF ablation has a primary role as a rhythm control strategy in the definite treatment of AF in patients with HF.

\section{Conclusion}

There should be a strong effort to prevent AF, since it would significantly improve the ability to deliver CRT in patients with heart failure. Because patients with slower heart rates are more likely to develop AF, a dual-chamber rate-modulated pacing mode (DDDR) may reduce interruptions of CRT. The search for better pharmacological maneuvers should continue to provide the help needed to cardiac devices. The incorporation of newer AF suppression algorhythm to CRT devices may be very useful in eliminating AF, allowing a better performance of the CRT device without interruption. Sinus rhythm is associated with a long-term improvement in left ventricular systolic function after $\mathrm{AF}$ ablation. Therefore, $\mathrm{AF}$ ablation may have a primary role as a rhythm control strategy in the definite treatment of $\mathrm{AF}$ in patients with $\mathrm{HF}$.

\section{Acknowledgments}

None.

\section{Conflicts of interest}

Authors declare that there is no conflict of interest.

\section{References}

1. Carson PE, Johnson GR, Dunkman WB, et al. The influence of atrial fibrillation on prognosis in mild to moderate heart failure: The V-HeFT studies Group. Circulation. 1993;87(6 Suppl):VI102-110.

2. Middlekauff HR, Stevenson WG, Stevenson LW. Prognostic significance of atrial fibrillation in advance heart failure: A study of 390 patients. Circulation. 1991;84(1):40-48.

3. Pozzoli M, Cioffi G, Traversi E, et al. Predictors of primary atrial fibrillation and concomitant clinical and hemodynamic changes in patients with chronic heart failure: A prospective study in 344 patients with baseline sinus rhythm. J Am Coll Cardiol. 1998;32(1):197-204. 
4. Dries DL, Exner DV, Gersh BJ, al. Atrial fibrillation is associated with an increased risk for mortality and heart failure progression in patients with asymptomatic and symptomatic left ventricular systolic dysfunction: a retrospective analysis of the SOLVD trials. Studies of Left Ventricular Dysfunction. J Am Coll Cardiol. 1988;32(3):695-703.

5. Bourassa MG, Gurne O, Bangdiwala SI, et al. Natural history and patterns of current practice in heart failure. J Am Coll Cardiol. 1993;22(4 Suppl A):14A-19A

6. Mathew J, Hunsberger S, Fleg J, et al. Incidence, predictive factors, and prognostic significance of supraventricular tachyarrhythmias in congestive heart failure. Chest. 2000;118(4):914-922.

7. Sipahi I, Fang JC. QRS duration criteria to select patients for cardiac resynchronization therapy: CRT should be reserved for a QRS duration $\geq 150$ ms: Pro. Circ Arrhythm Electrophysiol. 2013;6:436-442.

8. Turagam MK, Velagapudi P, Kocheril AG. Standardization of QRS duration measurement and LBBB criteria in CRT trials and Clinical Practice. Curr Cardiol Rev. 2013;9(1):20-23.

9. Fang F, Sanderson JE, Yu CM. Potential role of biventricular pacing beyond advanced systolic heart failure. Circ J. 2013;77(6):1364-1369.

10. Dai HL, Guang XF, Xiao ZC, et al. Application of echocardiography in resynchronization treatment of heart failure patients. Chin Med J. 2013;125(19):3548-3555.

11. Chen S, Yin Y, Krucoff MW. Effect of cardiac resynchronization therapy and implantable cardioverter defibrillator on quality of life in patients with heart failure: a meta-analysis. Europace. 2012;14(11):1602-1607.

12. Kannel WB, Abbott RD, Savage DD, et al. Epidemiologic features of chronic atrial fibrillation: The Framingham study. $N$ Engl J Med. 1982; 306(17):1018-1022.

13. Davies MJ, Pomerance A. Pathology of atrial fibrillation in man. $\mathrm{Br}$ Heart J. 1972;34(5):520-525.

14. Lev M. Aging changes in the human sinoatrial node. J Geront. 1954;9:1.

15. Davies MJ, Pomerance A. Quantitative study of aging changes in the human sinoatrial node and internodal tracts. Br Heart J. 1972;34(2):150 152 .

16. Hudson REB. The human pacemarker and its pathology. Br Heart J. $1960 ; 22: 153-167$

17. Centurion OA, Fukatani M, Konoe A, et al. Different distribution of abnormal endocardial electrograms within the right atrium in patients with sick sinus syndrome. Br Heart J. 1992;68(6):596-600.

18. Rensma PL, Allessie MA, Lammers WJ, et al. Length of excitation wave and susceptibility to reentrant atrial arrhythmias in normal conscious dogs. Circ Res. 1988;62(2):395-410.

19. Wiener N, Rosenblueth A. The mathematical formation of the problem of conduction of impulses in a network of connected excitable elements, specifically in cardiac muscle. Arch Inst Cardiol Mex. 1946;16(3):205265 .

20. Erickson EE, Lev M. Aging changes in the human AV node, bundle and bundle branches. J Gerontol.1952;7(1):1-12.

21. Spach MS, Dober PC, Anderson PA. Multiple regional differences in cellular properties that regulate repolarization and contraction in the right atrium of adult and newborn dogs. Circ Res. 1989;65(6):594-1611.

22. Spach MS, Dober PC. Relating extracellular potentials and their derivatives to anisotropic propagation at microscopic level in human cardiac muscle. Evidence for electrical uncoupling of side-to-side fiber connections with increasing age. Circ Res. 1986;58(3):356-371.
23. Escande D, Coulombe A, Faivre JF, et al. Two types of transient outward currents in adult human atrial cells. Am J Physiol. 1987;252(1 Pt 2):H142-H148.

24. Van wagoner DR, Pond AL, McCarthy PM, et al. Outward K+ current densities and $\mathrm{Kv} 1.5$ expression are reduced in chronic human atrial fibrillation. Circ Res.1987;80(6):772-781.

25. Fleg JL, Dhirenda ND, Lakatta EG. Right bundle branch block: Long term prognosis in apparently healthy men. $\mathrm{J} \mathrm{Am} \mathrm{Coll} \mathrm{Cardiol.}$ 1983;1(3):887-892.

26. Fleg JL, Kennedy HL. Cardiac arrhythmias in a healthy elderly population: detection by 24-hour ambulatory electrocardiography. Chest.1982;81(3):302-307.

27. Tresh DD, Fleg JL. Unexplained sinus bradycardia: Clinical significance and long-term prognosis in apparently healthy persons older than 40 years. Am J Cardiol. 1986;58(10):1009-1013.

28. Maurer MS, Shefrin EA, Fleg JL (1995) Prevalence and prognostic significance of exercise induced supraventricular tachycardia in apparently healthy volunteers. Am J Cardiol 75(12):788-792.

29. Centurion OA, Isomoto S, Shimizu A, et al. The effects of aging on atrial endocardial electrograms in patients with paroxysmal atrial fibrillation. Clin Cardiol. 2003;26(9):435-438.

30. Centurion OA, Shimizu A, Isomoto S, et al. Influence of advancing age on fractionated right atrial endocardial electrograms. Am J Cardiol. 2005;96(2):239-242.

31. Grines CL, Bashore TM, Boudoulas H, et al. Functional abnormalities in isolated left bundle branch block. The effect of inter ventricular asynchrony. Circulation. 1989;79(4):845-853.

32. Wyndham CR, Smith T, Meeran MK, et al. Epicardial activation in patients with left bundle branch block. Circulation.1980;61(4):696-703.

33. Takeshita A, Basta LL, Kioschos JM. Effect of intermittent left bundle branch block on left ventricular performance. Am J Med.1974;56(2):251255 .

34. Bramlet DA, Morris KG, Coleman RE, et al. Effect of rate-dependent left bundle branch block on global and regional left ventricular function. Circulation. 1983;67(5):1059-1065.

35. Kass DA, Chen $\mathrm{CH}$, Curry $\mathrm{C}$, et al. Improved left ventricular mechanics from acute VDD pacing in patients with dilated cardiomyopathy and ventricular conduction delay. Circulation. 1999;99(12):1567-1573.

36. Auricchio A, Stellbrink C, Block M, et al. Effect of pacing chamber and atrioventricular delay on acute systolic function of paced patients with congestive heart failure. The Pacing Therapies for Congestive Heart Failure Study Group. The Guidant Congestive Heart Failure Research Group. Circulation.1999;99(23):2993-3001.

37. Francis GS, Cohn JN, Johnson G, et al. Plasma norepinefrine, plasma renine activity, and congestive heart failure. Relations to survival and the effects of therapy in V-HeFT II. The V-HeFT VA Cooperative Studies Group. Circulation. 1993;87(6 Suppl):VI40-VI48.

38. Adamson PB, Kleckner KJ, VanHout WL, et al.Cardiac resynchronization therapy improves heart rate variability in patients with symptomatic heart failure. J Am Coll Cardiol.2003;108(3):266-269.

39. Yu CM, Chau E, Sanderson JE, Fan K, Tang MO, et al. Tissue Doppler echocardiographic evidence of reverse remodeling and improved synchronicity by simultaneously delaying regional contraction after biventricular pacing therapy in heart failure. Circulation. 2002;105(4):438-445. 
40. Knight BP, Desai A, Coman J, et al. Long-term retention of cardiac resynchronization therapy. J Am Coll Cardiol. 2004;44(1):72-77.

41. Vermes E, Tardif JC, Bourassa MG, et al. Enalapril decreases the incidence of atrial fibrillation in patients with left ventricular dysfunction Insight from the SOLVD study. Circulation. 2003;107(23):2926-2931.

42. Li D, Shinagawa K, Pang L, et al. Effects of angiotensin converting enzyme inhibition on the development of the atrial fibrillation substrate in dogs with ventricular tachypacing-induced congestive heart failure. Circulation. 2001;104(21):2608-2614.

43. Nakashima H, Kumagai K, Urata H, et al. Angiotensin II antagonist prevents electrical remodeling in atrial fibrillation. Circulation. 2000;101(22):2612-2617.

44. Madrid AH, Bueno MG, Rebollo JM, et al. Use of irbesartan to maintain sinus rhythm in patients with long-lasting persistent atrial fibrillation. Circulation. 2002;106(3):331-336.

45. Goette A, Arndt M, Rocken C, et al. Regulation of angiotensin II receptor subtypes during atrial fibrillation in humans. Circulation. 2000;101(23):2678-2681.

46. Wachtell K, Lehto M, Gerdts E, et al. Angiotensin II receptor blockade reduces new-onset atrial fibrillation and subsequent stroke compared to atenolol: The losartan intervention for end point reduction in hypertension (LIFE) study. J Am Coll Cardiol. 2005;45(5): 712-719.

47. Pedersen OD, Bagger H, Kober L, et al. Trandolapril reduces the incidence of atrial fibrillation after acute myocardial infarction in patients with left ventricular dysfunction. Circulation. 1999;100(4):376-380.

48. Wachtell K, Hornestam B, Lehto M, et al. Cardiovascular morbidity and mortality in hypertensive patients with a history of atrial fibrillation: The losartan intervention for end point reduction in hypertension (LIFE) study. J Am Coll Cardiol. 2005;45(5):705-711.

49. Gerdts E, Oikarinen L, Palmieri V, et al. Correlates of left atrial size in hypertensive patients with left ventricular hypertrophy: The losartan intervention for end point reduction in hypertension (LIFE) study. Hypertension. 2002;39(3):739-743.

50. Fuster V, Ryden LE, Asinger RW, et al. ACA/AHA/ESC guidelines for the management of patients with atrial fibrillation. J Am Coll Cardiol. 2001;38(4):1231-1265.

51. Benjamin EJ, D’Agostino RB, Belanger AJ, et al. Left atrial size and the risk of stroke and death. The Framingham heart study. Circulation. 1995;92(4):835-841.

52. Gottdiener JS, Reda DJ, Williams DW, et al. Effect of single-drug therapy on reduction ofleft atrial size in mild to moderatehypertension: Comparison of six antihypertensive agents. Circulation.1988;98(2):140-148.
53. Gauch P. Atrial fibrillation suppression algorithm in dual-chamber permanent pacemakers. Rev Soc Parag Cardiol. 2005;3:146-150.

54. Carlson MD, Ip J, Messenger J, Beau S, et al. A new pacemaker algorithm for the treatment of atrial fibrillation: Results of the Atrial Dynamic Overdrive Pacing Trial (ADOPT). J Am Coll Cardiol. 2003;42(4):27633.

55. Padeletti L. Atrial fibrillation in heart failure patients implanted with a cardiac resynchronization therapy device: One-year results of the randomized MASCOT study. American Heart Association Scientific Sessions, Orlando. 2007.

56. Padeletti L, Muto C, Maounis T, et al. Atrial fibrillation in recipients of cardiac resynchronization therapy device: 1 year results of the randomized MASCOT trial. Am Heart J. 2008;156(3):520-526.

57. Schuchert A, Muto C, Maounis T, et al. One year outcome after CRT implantation in NYHA class IV in comparison to NYHA class III patients. Clin Res Cardiol. 2013;102(7):505-511.

58. Dikinson O, Chen LY, Francis GS. Atrial fibrillation and heart failure: Intersecting population, morbidities and mortality. Heart Fail Rev. 2014;19(3):285-293

59. Arrigo M, Bettex D, Rindiger A. Management of atrial fibrillation in critically ill patients. Crit Care Res Pract. 2014:840615.

60. Khan MA, Ahmed F, Neyses L, et al. Atrial fibrillation in heart failure: The sword of Damocles revisited. World J Cardiol. 2013;5(7):215-227.

61. Silva-Cardoso J, Zharinov OJ, Ponikowski P, et al. Heart failure in patients with atrial fibrillation is associated with high symptom and hospitalization burden: The RealiseAF Survey. Clin Cardiol. 2013;36(12):766-774.

62. Nedios S, Sommer P, Dagres N, et al. Long-term follow-up after atria fibrillation ablation in patients with impaired left ventricular systolic function: The importance of rhythm and rate control. Heart Rhythm. 2014;11(3):344-351.

63. Heijman J, Voigt N, Abu-Taha IH, et al. Rhythm control of atrial fibrillation in heart failure. Heart Fail Clin. 2013;9(4):407-415.

64. Sairaku A, Nakano Y, Oda N, et al. Incomplete cure of tachycardiainduced cardiomyopathy secondary to rapid atrial fibrillation by heart rate control without sinus conversion. J Cardiovasc Electrophysiol. 2014;25(10):1037-1043.

65. Centurion OA. Atrial fibrillation complicating congestive heart failure: Electrophysiological aspects and its deleterious effect on cardiac resynchronization therapy. J Atrial Fib. 2009;1(7):417-429. 